Article

\title{
Microwave Assisted Synthesis of Novel Functionalized Hydantoin Derivatives and Their Conversion to 5-(Z) Arylidene-4H-imidazoles
}

\author{
Sukanta Kamila, Haribabu Ankati and Edward R. Biehl * \\ Department of Chemistry, Southern Methodist University, 3215 Daniel Avenue, Dallas, \\ TX 75275, USA
}

* Author to whom correspondence should be addressed; E-Mail: ebiehl@smu.edu;

Tel.: +1-214-768-2735; Fax: +1-214-768-4089.

Received: 24 May 2011; in revised form: 22 June 2011 / Accepted: 27 June 2011 /

Published: 29 June 2011

\begin{abstract}
Alkyl-1-yl)-1H-imidazol-5(4H)-ones 5a-n were synthesized via nucleophilic substitution of the methylsulfanyl group of the corresponding 2-(methylthio)-1H-imidazol5(4H)-ones 3a-c with suitably substituted secondary amines. The starting 2-thioxoimidazolidin-4-ones $\mathbf{2 a}$,2b were prepared by condensation of thiohydantoin and benzo[b]thiophene-3-carbaldehyde or benzofuran-3-carbaldehyde under microwave irracdiation (MW) conditions. 2-Methylthio derivatives 3a-c were prepared by treatment of $\mathbf{2 a - b}$ with methyl iodide in the presence of aqueous sodium hydroxide.
\end{abstract}

Keywords: MW assisted synthesis; hydantoin; arylidene- $4 H$-imidazoles

\section{Introduction}

Hydantoin derivatives have achieved considerable success as anticonvulsant agents [1]. The nucleosides of several 5-arylidene-3-arylhydantoins and 2-thiohydantoins show potent activity against human immunodeficiency virus (HIV) [2] and the leukemia subpanel [3]. A recent study showed that S-glucosylated hydantoins (Figure 1) act against herpes simplex virus, type 1 (HSV-1) and type 2 (HSV-2) in Vero cells [4]. A further study reported that thiazolidinediones (TZDs), which are known to have potent enhancing effects on insulin sensitivity, have been developed for the treatment of noninsulin-dependent diabetes mellitus $[5,6]$. 
Figure 1. Chemical structure of several biologically important compounds.

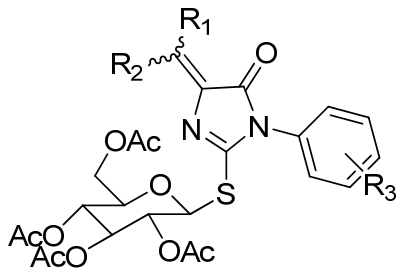

S-glucosylated hydantoins<smiles>Cc1c(C)c2c(c(C)c1O)CCC(C)(COc1ccc(CC3SC(=O)NC3=O)cc1)O2</smiles>

Troglitazone<smiles>[Y]=c1[nH]c(=O)/c(=C/c2cc[R1]cc2)s1</smiles>

2,4-Thiazolidinedione derivatives

It also been found that TZDs are high-affinity ligands for peroxisome proliferitor-activated receptor- $\gamma($ PPAR- $\gamma$ ) and inhibit the production of monocyte chemo attractant protein 1 (MCP-1) in some human tissues [6]. Another group of TZDs revealed that troglitazone (Figure 1) acts on acute liver injury induced by ethanol and lipopolysaccharide [7]. For the past few years our group has been working on biologically important compounds [8-10]. Herein we report on the synthesis of variety of benzo $[b]$ thiophene and benzofuran based hydantoin derivatives with the aim of investigating their antimicrobial and neuroprotecting properties.

\section{Results and Discussion}

As shown in Scheme 1, various substituted hydantion derivatives $\mathbf{5 a - n}$ were prepared by the condensation between benzo[b]thiophene-3-carbaldehyde and benzofuran-3-carbaldehyde with thiohydantoin using MW irradiation and a catalytic amount of 2,2,6,6-tetramethyl piperidine in ethanol that yielded (Z)-5-(benzo[b]thiophen-3-ylmethylene)-2-thioxoimidazolidin-4-one (2a) and (Z)-5(benzofuran-3-ylmethylene)-2-thioxoimidazolidin-4-one (2b), respectively.

Scheme 1. Schematic representation for the synthesis of compounds $\mathbf{5 a - n}$.<smiles>[X]c1ccccc1C=O</smiles>

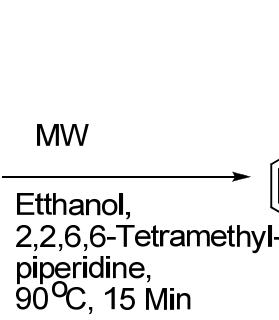

piperidine,
$90^{\circ} \mathrm{C}, 15 \mathrm{Min}$<smiles>[X]c1ccccc1-c1ccccc1</smiles>

$2 a, x=S$ $2 b, X=0$<smiles>[X]c1ccccc1/C=C1\N=C(SC)N([R])C1=O</smiles>

3a, $X=s, R=H$

$3 b, X=s, R=\mathrm{CH}_{3}$

$3 c, X=0, R=\mathrm{CH}_{3}$<smiles>[Z][Y]1CC([R])NC([R])C([Y9])C1[R3]</smiles><smiles>[R6][Y]1cc(C=C2N=C(N3C(=O)C=C(c4ccccc4[R16])C3[R])C([R])C2[R])c2ccccc12</smiles>

5a-n

$\mathrm{R}=\mathrm{H}, \mathrm{CH}_{3}$

$\mathbf{R}_{\mathbf{1}}=\mathbf{R}_{\mathbf{2}}=\mathrm{H}, \mathrm{CH}_{\mathbf{3}}$

$\mathbf{R}_{3}=\mathrm{H}, \mathrm{CH}_{3}$

$\mathrm{Y}=\mathrm{CH}_{2}, \mathrm{O}$ 
Since the starting material for $\mathbf{2 b}$ is not commercially available, it was prepared according to the literature procedure [11,12] which involved converting 2-hydroxy acetophenone to 3-methylbenzofuran [11] which was subsequently transformed to benzofuran-3-carbaldehyde by refluxing with $\mathrm{SeO}_{2}$ in 1,4-dioxane [12].

Although many bases shown in Table 1 can be used as catalysts [e.g., piperidine, pyridine, $N$-methylpiperidine (NMP), DBU)], 2,2,6,6-Tetramethylpiperidine (TMP) works best. The same reaction under conventional reflux condition using ethanol as solvent gave lower yields after longer times $(5 \mathrm{~h})$ and/or compounds that required rigorous purification. However the MW reaction provides cleaner reactions, shorter times $(15 \mathrm{~min})$ and purification of the products only required washing with cold ethanol. In addition the yields are good to excellent. The optimum temperature and condition for this MW assisted reaction was determined by a series of reactions of appropriate aldehyde $\mathbf{1}$ with hydantoin. The results are summarized in Table 1 . The results indicate that $\mathrm{MW}$ irradiation at $90{ }^{\circ} \mathrm{C}$ for $15 \mathbf{m i n}$ in ethanol is the optimum condition for the synthesis of $\mathbf{2} \mathbf{a}$ and $\mathbf{2} \mathbf{b}$.

Table 1. Screening of solvents, reaction time and temperature for the synthesis of $\mathbf{2 a}$.

\begin{tabular}{|c|c|c|c|c|c|}
\hline Entry & Base & Condition $^{\mathrm{a}}$ & Temp. $\left({ }^{\circ} \mathrm{C}\right)$ & Time (min) & Yield (\%) ${ }^{b}$ \\
\hline 1 & - & No solvent & 90 & 15 & trace \\
\hline 2 & - & Ethanoll & 90 & 15 & trace \\
\hline 3 & Piperidine & Ethanol & 90 & 15 & 80 \\
\hline 4 & TMP & Ethanol & 90 & 15 & 96 \\
\hline 5 & TMP & No solvent & 90 & 15 & trace \\
\hline 6 & DBU/Pyridine & Ethanol & 90 & 20 & 20 \\
\hline 7 & TMP & Acetonitrile & 90 & 15 & 76 \\
\hline 8 & TMP & Acetonitrile & 130 & 15 & 15 \\
\hline 9 & DBU & Acetonitrile & 90 & 30 & trace \\
\hline 10 & TMP & DMF & 90 & 15 & 45 \\
\hline 11 & NMP & $\mathrm{DMF}$ & 90 & 30 & 10 \\
\hline 12 & DBU & $\mathrm{DMF}$ & $120-140$ & 15 & trace \\
\hline 13 & TMP & Water & 90 & 15 & trace \\
\hline 14 & TMP & Water & 130 & 15 & trace \\
\hline 15 & - & water & 130 & 30 & trace \\
\hline 16 & TMP & Tolune & 90 & 15 & trace \\
\hline 17 & TMP & Isopropanol & 90 & 15 & 45 \\
\hline 18 & TMP & THF & 90 & 15 & 38 \\
\hline 19 & TMP & n-Butanol & 90 & 15 & 33 \\
\hline
\end{tabular}

The $Z$ configuration of the compounds $\mathbf{2 a}$ and $\mathbf{2} \mathbf{b}$ was confirmed by comparison of previously reported [2] hydantoin derivatives along with nuclear Overhauser effect (NOE) experiments [4]. The vinylic proton in the ${ }^{1} \mathrm{H}-\mathrm{NMR}$ spectra of benzo[b]thiophene and benzofuran derivatives appeared as singlets at $6.72 \mathrm{ppm}$ and $6.58 \mathrm{ppm}$, respectively. The intermediates $\mathbf{2 a}$ and $\mathbf{2 b}$ were in turn transformed into the corresponding methylsulfanyl derivatives $3(\mathbf{a}-\mathbf{c})$ by alkylation with methyl iodide in basic medium [13]. The use of 1.3 equiv. of alkyl halide and stirring for $4 \mathrm{~h}$ afforded 3a while use of excess 
alkylating agent (2.5 equiv.) and stirring for $16 \mathrm{~h}$ afforded dialkylation giving $3 \mathbf{b}$ in high yield. The ${ }^{13} \mathrm{C}-\mathrm{NMR}$ spectrum of compounds 3a-c showed the absence of $\mathrm{C}=\mathrm{S}$ signal of $\mathbf{2 a}$, $\mathbf{2} \mathbf{b}$ at $179.50 \mathrm{ppm}$ and the appearance of the $\mathrm{C}=\mathrm{N}$ signal at $170.9 \mathrm{ppm}$, corresponding to s-alkylation. On the other hand, the ${ }^{1} \mathrm{H}-\mathrm{NMR}$ spectra of $\mathbf{2 a}$ and $\mathbf{2 b}$ showed two NH signals at 12.41 and 12.18 which were absent in the spectra of 3a and $\mathbf{3 b}$, in which only a broad peak NH signal at 11.84 ppm appeared. Conversion of 2a to 3a via microwave irradiation was unsuccessful. The intermediate methylsulfanyl derivatives were subsequently converted into compounds $\mathbf{5 a - n}$ by nucleophilic substitution of the methylsulfanyl group with suitably substituted secondary amines [14] by using MW irradiation of a solution containing excess amine (>12.5 equiv.) and absolute ethanol at an elevated temperature $\left(100{ }^{\circ} \mathrm{C}\right)$. The same reaction under conventional reflux condition using ethanol as solvent required longer time $(16 \mathrm{~h})$ and/or rigorous purification of products As shown in Table 2, the 5-(Z) arylidene- $4 \mathrm{H}$-imidazole compounds 5a-n were formed in good to excellent yields. In most cases, the 5-(Z) arylidene- $4 H_{-}$ imidazoles precipitated upon cooling the reaction mixture and only simple trituration with ethanolhexane followed by recrystallization from ethanol afforded the product $\mathbf{5 a - 1}$. But in case of $\mathbf{5 m}$ and $\mathbf{5 n}$, the crude reaction mixtures were purified by column chromatography using $40 \%$ ethyl acetate-hexane mixture (v/v). All the products were well characterized by ${ }^{1} \mathrm{H}-\mathrm{NMR},{ }^{13} \mathrm{C}-\mathrm{NMR}$, IR and HRMS.

Table 2. MW assisted synthesis of various 5-(Z) arylidene- $4 H$-imidazoles.

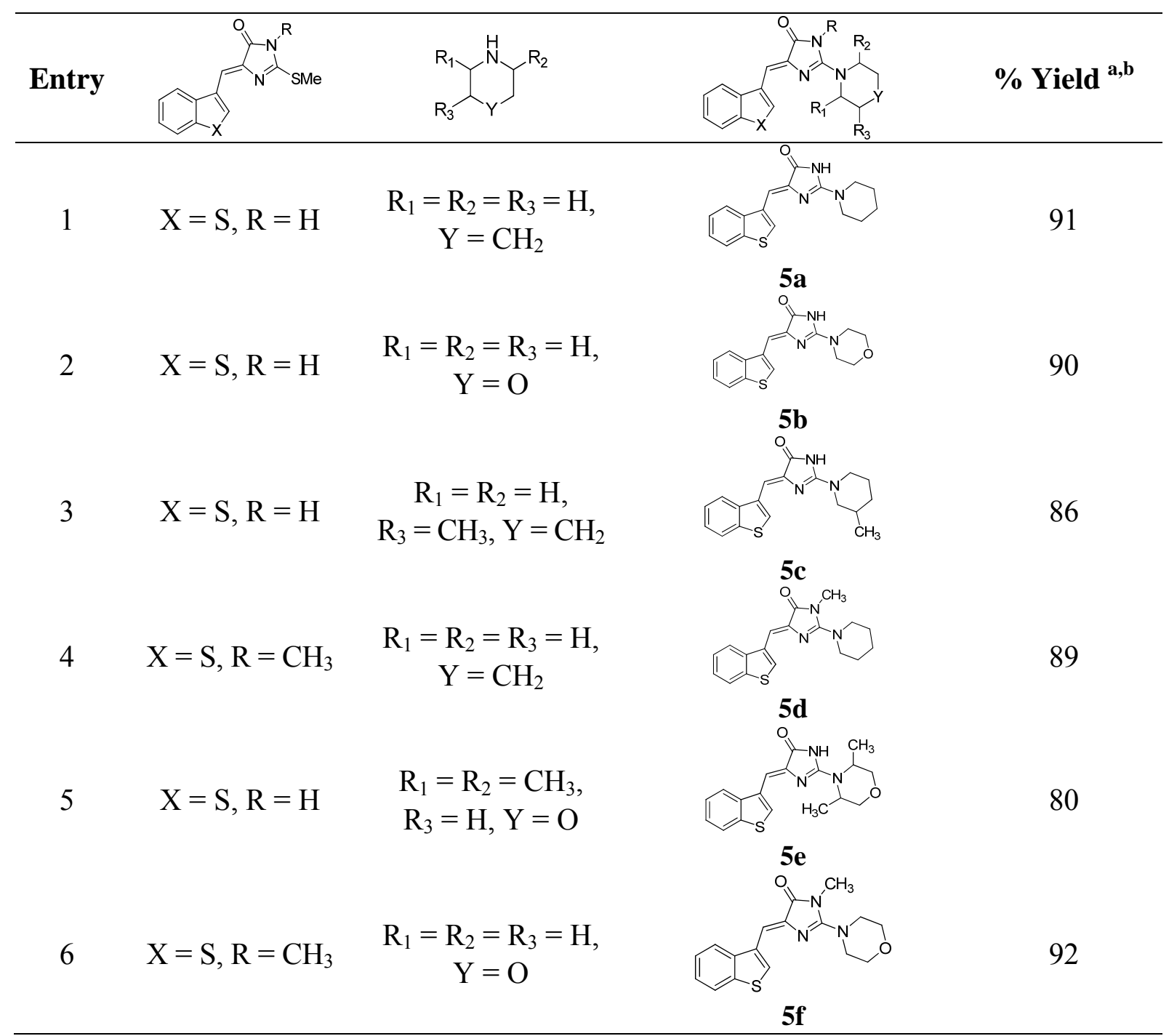


Table 2. Cont.

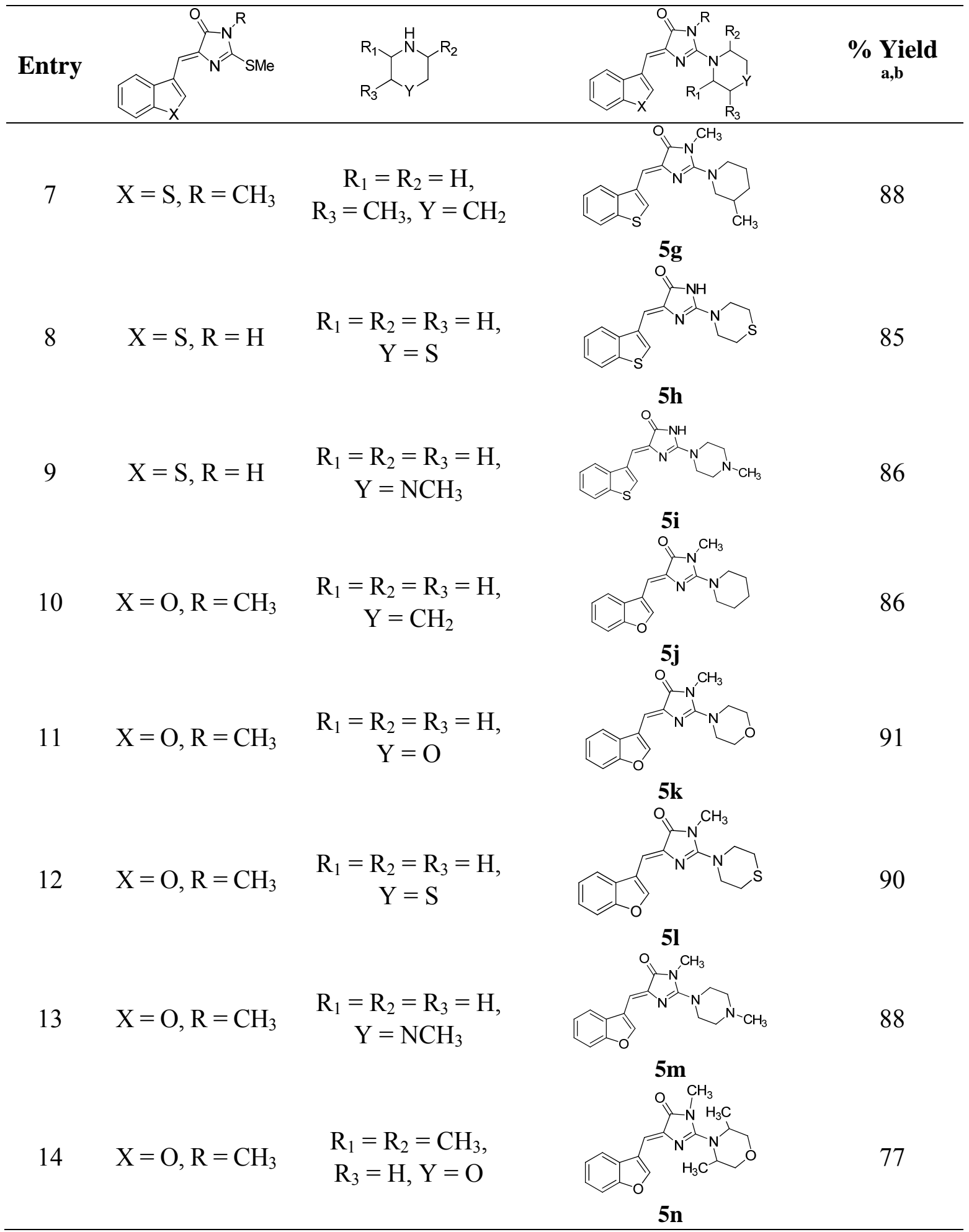

${ }^{a}$ Isolated yield; ${ }^{b}$ All the products were characterized by IR, ${ }^{1} \mathrm{H}-\mathrm{NMR},{ }^{13} \mathrm{C}-\mathrm{NMR}, \mathrm{DEPT}, \mathrm{HRMS}$ and elemental analysis.

The use of aromatic amines for the same reaction was unsuccessful. From Table 1, it can be seen that in the case of 3,5-dimethylmorpholine (entry 14) the comparatively low yield probably reflects unfavorable steric interaction between the two methyl groups. 


\section{Experimental}

\section{General}

The ${ }^{1} \mathrm{H}$ - and ${ }^{13} \mathrm{C}-\mathrm{NMR}$ spectra were recorded on a $500-\mathrm{MHz}$ Jeol multinuclear NMR spectrometer; chemical shifts were referenced to tetramethylsilane (TMS) as internal standard. Infrared (IR) spectra were obtained on a Varian 3100 Fourier transform (FT)-IR Spectrometer. Melting points were taken on a Meltemp apparatus. All chemicals and reagents were purchased from commercial sources. Mass spectra were obtained from Washington University, St. Louis. MO. Microwave experiments were carried out on CEM Discover microwave instrument.

(Z)-5-(Benzo[b] thiophen-3-ylmethylene)-2-thioxoimidazolidin-4-one (2a) under MW irradiation. An equimolar quantity of benzo[b]thiophene-3-carbaldehyde and thiohydantoin were mixed in a mortar pestle and charged into a specially designed MW test tube. After the addition of ethanol $(2 \mathrm{~mL})$ followed by 1-2 drops of 2,2,6,6-tetramethylpiperidine, the test tube was sealed then irradiated for 15 min at $90^{\circ} \mathrm{C}$ and 150 psi pressure. After cooling, the solid mass was scraped out of the test tube and placed into a flask containing $95 \%$ ethanol $(20 \mathrm{~mL})$ and filtered. The solid mass was collected then washed with ethanol $(20 \mathrm{~mL})$ and dried under reduced pressure and the residue was recrystallized from ethanol to afford $0.49 \mathrm{~g}(96 \%)$ of (Z)-5-(benzo[b]thiophen-3-ylmethylene)-2-thioxoimidazolidin-4-one (2a) as yellow floppy crystals. m.p.: 259-261 ${ }^{\circ} \mathrm{C}$; IR $(\mathrm{KBr}) \vee\left(\mathrm{cm}^{-1}\right)$ : 3274, 1723, 1649, 1496; ${ }^{1} \mathrm{H}-\mathrm{NMR}$ (DMSO-d $\mathrm{d}_{6}$ ): $\delta: 12.41$ (brs, $\left.1 \mathrm{H}, \mathrm{NH}\right), 12.18$ (brs, $\left.1 \mathrm{H}, \mathrm{NH}\right), 8.49$ (s, $\left.1 \mathrm{H}, \mathrm{Ar}-\mathrm{CH}\right), 8.02$ (dd, $J=7.45 \mathrm{~Hz}, 8.00 \mathrm{~Hz}, 2 \mathrm{H}, \mathrm{Ar}-\mathrm{CH}), 7.46-7.42(\mathrm{~m}, 2 \mathrm{H}, \mathrm{Ar}-\mathrm{CH}), 6.72$ (s, 1H, vinylic proton); ${ }^{13} \mathrm{C}-\mathrm{NMR}$ $\left(\mathrm{DMSO}_{-} \mathrm{d}_{6}\right) \delta: 179.5(\mathrm{C}), 166.1(\mathrm{C}), 139.1(\mathrm{C}), 138.4(\mathrm{C}), 129.9(\mathrm{CH}), 129.0(\mathrm{C}), 127.8(\mathrm{C}), 125.7(\mathrm{CH})$, $125.6(\mathrm{CH}), 123.6(\mathrm{C}), 122.0(\mathrm{CH}), 102.3(\mathrm{CH})$; HRMS m/z: 261.0161 found (calculated for $\mathrm{C}_{12} \mathrm{H}_{8} \mathrm{~N}_{2} \mathrm{O}_{2} \mathrm{~S}_{2},[\mathrm{M}+\mathrm{H}]^{+}$requires 261.0158).

(Z)-5-(Benzofuran-3-ylmethylene)-2-thioxoimidazolidin-4-one (2b). Following the procedure for the preparation of 2a, the microwave-assisted reaction of equimolar quantitites of benzofuran-3carbaldehyde and thiohydantoin gave $0.51 \mathrm{~g}(91 \%)$ of $\mathbf{2 b}$ as a dark brown solid. m.p.: $307-308{ }^{\circ} \mathrm{C}$; IR (KBr) $v\left(\mathrm{~cm}^{-1}\right): 3209,1725,1648,1484,1452 ;{ }^{1} \mathrm{H}-\mathrm{NMR}\left(\mathrm{DMSO}-d_{6}\right) \delta$ : 12.39 (brs, $\left.1 \mathrm{H}, \mathrm{NH}\right), 11.95$ (brs, 1H, NH), $8.83(\mathrm{~s}, 1 \mathrm{H}, \mathrm{Ar}-\mathrm{CH}), 7.88(\mathrm{~d}, J=8 \mathrm{~Hz}, 1 \mathrm{H}, \mathrm{Ar}-\mathrm{CH}), 7.61(\mathrm{~d}, J=8 \mathrm{~Hz}, 1 \mathrm{H}, \mathrm{Ar}-\mathrm{CH})$, 7.38-7.30 (m, 2H, Ar-CH), 6.58 (s, 1H, vinylic proton); ${ }^{13} \mathrm{C}-\mathrm{NMR}$ (DMSO-d $\left.{ }_{6}\right) \delta: 179.2(\mathrm{C}), 165.7(\mathrm{C})$, $154.7(\mathrm{C}), 147.1(\mathrm{CH}), 128.7(\mathrm{C}), 126.6(\mathrm{C}), 125.9(\mathrm{CH}), 124.1(\mathrm{CH}), 120.3(\mathrm{CH}), 114.3(\mathrm{CH}), 112.0$ (CH), $99.8(\mathrm{CH})$; HRMS m/z: 245.0390 found (calculated for $\mathrm{C}_{12} \mathrm{H}_{8} \mathrm{~N}_{2} \mathrm{OS}_{2},[\mathrm{M}+\mathrm{H}]^{+}$requires 245.0386).

(Z)-4-(Benzo[b]thiophen-3-ylmethylene)-2-(methylthio)-1H-imidazol-5(4H)-one (3a). Ethyl iodide $(2.15 \mathrm{~g}, 15.1 \mathrm{mmol})$ was added to a solution of $2 \mathrm{a}(2.9 \mathrm{~g}, 11.1 \mathrm{mmol})$, in $15 \%$ aqueous $\mathrm{NaOH}(4.5 \mathrm{~mL})$ and $95 \%$ methanol $(30 \mathrm{~mL})$ and the resulting reaction mixture was stirred for $4 \mathrm{~h}$ at room temperature. Most of the solvent was removed by distillation and to the precipitate, was added $15 \mathrm{~mL}$ of water and stirred for $10 \mathrm{~min}$ and filtered and washed first with water $(200 \mathrm{~mL})$ and then with diethyl ether $(50 \mathrm{~mL})$ to supply $2.85 \mathrm{~g}$ of $(\mathrm{Z})-4$-(benzo[ $b]$ thiophen-3-ylmethylene)-2-(methylthio)-1H-imidazol-5(4H)-one (3a) as a bright yellow solid. m.p.: 276-278 ${ }^{\circ} \mathrm{C}$; IR $(\mathrm{KBr}) v\left(\mathrm{~cm}^{-1}\right)$ : 3058, 1701, 1627, 1555, 1492; ${ }^{1} \mathrm{H}-\mathrm{NMR}\left(\mathrm{DMSO}-\mathrm{d}_{6}\right) \delta$ : 11.84 (brs, 1H, NH), 8.94 (s, 1H, Ar-CH), 8.11 (d, J=7.5 Hz, 1H, Ar-CH), 
$8.01(\mathrm{~d}, J=8 \mathrm{~Hz}, 1 \mathrm{H}, \mathrm{Ar}-\mathrm{CH}), 7.44-7.40(\mathrm{~m}, 2 \mathrm{H}, \mathrm{Ar}-\mathrm{CH}), 7.04$ (s, 1H, vinylic proton), 2.66 (s, 3H, $\left.\mathrm{SCH}_{3}\right) ;{ }^{13} \mathrm{C}-\mathrm{NMR}\left(\mathrm{DMSO}_{6}\right) \delta: 170.9(\mathrm{C}), 165.9(\mathrm{C}), 140.2(\mathrm{C}), 139.4(\mathrm{C}), 138.5(\mathrm{C}), 133.0(\mathrm{CH})$, $130.1(\mathrm{C}), 125.4(\mathrm{CH}), 123.5 \mathrm{CH}), 121.9(\mathrm{CH}), 111.2(\mathrm{CH}), 12.9\left(\mathrm{SCH}_{3}\right) ; \mathrm{HRMS} \mathrm{m} / \mathrm{z}: 275.0322$ found (calculated for $\mathrm{C}_{13} \mathrm{H}_{10} \mathrm{~N}_{2} \mathrm{OS}_{2},[\mathrm{M}+\mathrm{H}]^{+}$requires 275.0315).

(Z)-4-(Benzo[b]thiophen-3-ylmethylene)-1-methyl-2-(methylthio)-1H-imidazol-5(4H)-one (3b). Compound 3b was prepared in same manner as 3a with the exception that an excess (2.5 equiv.) of methyl iodide was used and stirring was carried out for $16 \mathrm{~h}$. This reaction afforded $2.9 \mathrm{~g}(90 \%$ yield $)$ of $\mathbf{3 b}$ as a light brown solid. m.p.: $260-263{ }^{\circ} \mathrm{C}$; IR $(\mathrm{KBr}) \vee\left(\mathrm{cm}^{-1}\right)$ : 1700, 1643, 1540, 1497; ${ }^{1} \mathrm{H}-\mathrm{NMR}\left(\mathrm{DMSO}-d_{6}\right) \delta$ : 8.99 (s, 1H, Ar-CH), $8.16(\mathrm{~d}, J=7.5 \mathrm{~Hz}, 1 \mathrm{H}, \mathrm{Ar}-\mathrm{CH}), 8.03$ (d, J=7.5 Hz, 1H, Ar-CH), 7.46-7.41 (m, 2H, Ar-CH), 7.14 (s, 1H, vinylic proton), $3.06\left(\mathrm{~s}, 3 \mathrm{H}, \mathrm{NCH}_{3}\right), 2.72\left(\mathrm{~s}, 3 \mathrm{H}, \mathrm{SCH}_{3}\right) ;{ }^{13} \mathrm{C}-\mathrm{NMR}$ $\left(\mathrm{DMSO}_{6}\right) \delta$ : $169.1(\mathrm{C}), 166.9(\mathrm{C}), 139.4(\mathrm{C}), 139.1$ (C), 138.5 (C), $\left.133.6(\mathrm{CH}), 130.0 \mathrm{C}\right), 125.5$ $(\mathrm{CH}), 123.5(\mathrm{CH}), 122.1(\mathrm{CH}), 112.4(\mathrm{CH}), 26.9\left(\mathrm{CH}_{3}\right), 13.2\left(\mathrm{CH}_{3}\right)$; HRMS m/z: 289.0483 found (calculated for $\mathrm{C}_{14} \mathrm{H}_{12} \mathrm{~N}_{2} \mathrm{OS}_{2},[\mathrm{M}+\mathrm{H}]^{+}$requires 289.0471).

(Z)-4-(Benzofuran-3-ylmethylene)-1-methyl-2-(methylthio)-1H-imidazol-5(4H)-one (3c). Compound 3c was obtained as a brown solid in $89 \%$ when prepared by the same method used for the preparation of 3a. m.p.: $168-171{ }^{\circ} \mathrm{C}$; IR (KBr) 1701, 1642, 1541, $1496 \mathrm{~cm}^{-1} ;{ }^{1} \mathrm{H}$ NMR (DMSO-d $\left.)_{6}\right): \delta 8.73(\mathrm{~s}, 1 \mathrm{H}$, $\operatorname{Ar}-\mathrm{CH}), 8.29$ (d, $J=7.45 \mathrm{~Hz}, 1 \mathrm{H}, \mathrm{Ar}-\mathrm{CH}), 7.60$ (d, J = 7.45 Hz, 1H, Ar-CH, 7.35-7.29 (m, 2H, $\mathrm{Ar}-\mathrm{CH}), 7.05$ (s, $1 \mathrm{H}$, vinylic proton), $3.03\left(\mathrm{~s}, 3 \mathrm{H}, \mathrm{NCH}_{3}\right), 2.70\left(\mathrm{~s}, 3 \mathrm{H}, \mathrm{SCH}_{3}\right) ;{ }^{13} \mathrm{C}-\mathrm{NMR}$ (DMSO-d $\left.\mathrm{d}_{6}\right)$ $\delta: 168.5(\mathrm{C}), 165.7(\mathrm{C}), 155.2(\mathrm{C}), 150.4(\mathrm{CH}), 138.7(\mathrm{C}), 126.1(\mathrm{C}), 125.8(\mathrm{CH}), 124.0(\mathrm{CH}), 121.9$ $(\mathrm{CH}), 117.0(\mathrm{C}), 112.3(\mathrm{CH}), 112.0(\mathrm{CH}), 26.8\left(\mathrm{CH}_{3}\right), 13.2\left(\mathrm{CH}_{3}\right)$; HRMS m/z: 273.0701 found (calculated for $\mathrm{C}_{14} \mathrm{H}_{12} \mathrm{~N}_{2} \mathrm{O}_{2} \mathrm{~S}$, $[\mathrm{M}+\mathrm{H}]^{+}$requires 273.0699).

(Z)-4-(Benzo[b]thiophen-3-ylmethylene)-2-(piperidin-1-yl)-1H-imidazol-5(4H)-one (5a). Compound 3a and piperidine (excess, 12.5 equiv.) were mixed then placed in a specially designed MW test tube. Ethanol $(2 \mathrm{~mL})$ was added to the mixture. The test tube was then sealed and then irradiated for $30 \mathrm{~min}$ at $100{ }^{\circ} \mathrm{C}$ and 150 psi pressure. After cooling, the solid mass was filtered and crashed into $20 \mathrm{~mL}$ of $95 \%$ ethanol. The solid mass collected was washed with ethanol-hexane mixture $(1: 5 \mathrm{v} / \mathrm{v})$ and dried over vacuum to get the desired product. The titled compound was obtained as bright yellow solid. m.p.: $275-277{ }^{\circ} \mathrm{C}$; IR (KBr) $v\left(\mathrm{~cm}^{-1}\right)$ : 3111.7, 1695, 1643, 1588; ${ }^{1} \mathrm{H}-\mathrm{NMR}$ (DMSO-d $\mathrm{d}_{6}$ ) $\delta$ : 11.20 (brs, 1H, NH), 8.67 (s, 1H, Ar-CH), 7.98 (dd, J=4.5 Hz, 7.4 Hz, 2H, Ar-CH), 7.42-7.37 (m, 2H, Ar-CH), 6.63 (s, $1 \mathrm{H}$, vinylic proton), 3.59 (brs, $\left.4 \mathrm{H}, \mathrm{CH}_{2} \times 2\right), 1.58-1.55\left(\mathrm{~m}, 6 \mathrm{H}, \mathrm{CH}_{2} \times 3\right) ;{ }^{13} \mathrm{C}-\mathrm{NMR}\left(\mathrm{DMSO}-d_{6}\right)$

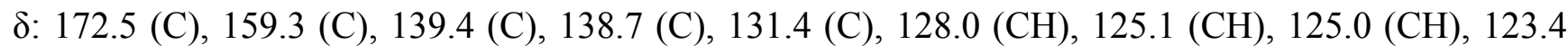
$(\mathrm{CH}), 121.7(\mathrm{CH}), 101.5(\mathrm{CH}), 45.0\left(\mathrm{NCH}_{2}\right), 25.7\left(\mathrm{CH}_{2}\right), 24.1\left(\mathrm{CH}_{2}\right) ; \mathrm{HRMS} \mathrm{m} / \mathrm{z}: 312.1173$ found (calculated for $\mathrm{C}_{17} \mathrm{H}_{17} \mathrm{~N}_{3} \mathrm{OS}$, $[\mathrm{M}+\mathrm{H}]^{+}$requires 312.1165 ) All the compounds $5 \mathbf{b}-\mathbf{n}$ were prepared in the same way.

(Z)-4-(Benzo[b]thiophen-3-ylmethylene)-2-morpholino-1H-imidazol-5(4H)-one (5b). This compound was obtained as light yellow solid. m.p.: 292-294 ${ }^{\circ} \mathrm{C}$; IR $(\mathrm{KBr}) \vee\left(\mathrm{cm}^{-1}\right): 3140,1686,1648,1591$,

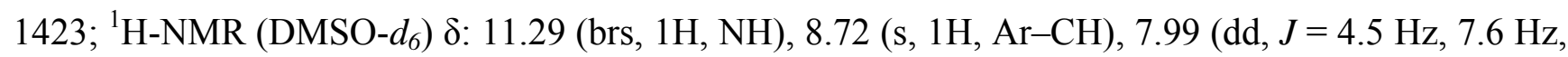
$2 \mathrm{H}, \mathrm{Ar}-\mathrm{CH}), 7.42-7.37$ (m, 2H, Ar-CH), 6.69 (s, 1H, vinylic proton), 3.67-3.62 (m, 8H, $\left.\mathrm{CH}_{2} \times 4\right)$; ${ }^{13} \mathrm{C}-\mathrm{NMR}\left(\mathrm{DMSO}-d_{6}\right) \delta$ : $172.2(\mathrm{C}), 159.4(\mathrm{C}), 139.4(\mathrm{C}), 138.7(\mathrm{C}), 131.2(\mathrm{C}), 128.5(\mathrm{CH}), 125.2(\mathrm{CH})$, 
$123.5(\mathrm{CH}), 121.8(\mathrm{CH}), 102.7(\mathrm{CH}), 66.1\left(\mathrm{OCH}_{2}\right), 40.3\left(\mathrm{NCH}_{2}\right) ; \mathrm{HRMS} \mathrm{m} / \mathrm{z}: 314.0963$ found (calculated for $\mathrm{C}_{16} \mathrm{H}_{15} \mathrm{~N}_{3} \mathrm{O}_{2} \mathrm{~S}$, [M+H] $]^{+}$requires 314.0957).

(Z)-4-(Benzo[b] thiophen-3-ylmethylene)-2-(3-methylpiperidin-1-yl)-1H-imidazol-5(4H)-one (5c). This compound was obtained as white solid. m.p.: $242-244{ }^{\circ} \mathrm{C}$; IR $(\mathrm{KBr}) v\left(\mathrm{~cm}^{-1}\right): 3206,1692,1616,1426$, 1407; ${ }^{1} \mathrm{H}-\mathrm{NMR}\left(\mathrm{DMSO}-d_{6}\right.$ ) $\delta: 11.15$ (brs, $\left.1 \mathrm{H}, \mathrm{NH}\right), 8.66$ (s, $\left.1 \mathrm{H}, \mathrm{Ar}-\mathrm{CH}\right), 7.99$ (dd, J=4.3 Hz, 7.9 Hz, $2 \mathrm{H}, \mathrm{Ar}-\mathrm{CH}), 7.42-7.37(\mathrm{~m}, 2 \mathrm{H}, \mathrm{Ar}-\mathrm{CH}), 6.62$ (s, 1H, vinylic proton), 4.25 (brs, $2 \mathrm{H}, \mathrm{CH}_{2}$ ), 3.01 (dd, $\left.J=10 \mathrm{~Hz}, 10.5 \mathrm{~Hz}, 1 \mathrm{H}, \mathrm{CH}_{2}\right), 2.70$ (brs, $\left.1 \mathrm{H}, \mathrm{CH}_{2}\right), 1.76-1.44\left(\mathrm{~m}, 4 \mathrm{H}, \mathrm{CH}_{2} \times 2\right), 1.17-1.13(\mathrm{~m}, 1 \mathrm{H}$, $\mathrm{CH}), 0.87$ (d, $\left.J=4.5 \mathrm{~Hz}, 3 \mathrm{H}, \mathrm{CH}_{3}\right) ;{ }^{13} \mathrm{C}-\mathrm{NMR}\left(\mathrm{DMSO}_{6}\right) \delta: 172.3$ (C), 159.5 (C), 139.4 (C), 138.7 (C), $131.4(\mathrm{C}), 127.9(\mathrm{CH}), 125.1(\mathrm{CH}), 125.0(\mathrm{CH}), 123.4 \mathrm{CH}), 121.7(\mathrm{CH}), 101.4(\mathrm{CH}), 51.2\left(\mathrm{NCH}_{2}\right)$, $45.3\left(\mathrm{NCH}_{2}\right), 32.6\left(\mathrm{CH}_{2}\right), 31.0(\mathrm{CH}), 24.9\left(\mathrm{CH}_{2}\right), 18.9\left(\mathrm{CH}_{3}\right)$; HRMS m/z: 326.1334 found (calculated for $\mathrm{C}_{18} \mathrm{H}_{19} \mathrm{~N}_{3} \mathrm{OS},[\mathrm{M}+\mathrm{H}]^{+}$requires 326.1322).

(Z)-4-(Benzo[b]thiophen-3-ylmethylene)-1-methyl-2-(piperidin-1-yl)-1H-imidazol-5(4H)-one (5d). This compound was obtained as light yellow solid. m.p.: $165-168^{\circ} \mathrm{C}$; IR $(\mathrm{KBr}) v\left(\mathrm{~cm}^{-1}\right)$ : 1713, 1638, 1560, 1451; ${ }^{1} \mathrm{H}-\mathrm{NMR}\left(\mathrm{DMSO}_{6}\right) \delta: 8.77$ (s, $\left.1 \mathrm{H}, \mathrm{Ar}-\mathrm{CH}\right), 8.02$ (dd, $\left.J=7.2 \mathrm{~Hz}, 7.8 \mathrm{~Hz}, 2 \mathrm{H}, \mathrm{Ar}-\mathrm{CH}\right)$, 7.43-7.38 (m, 2H, Ar-CH), $6.84\left(\mathrm{~s}, 1 \mathrm{H}\right.$, vinylic proton), $3.55\left(\mathrm{~s}, 4 \mathrm{H}, \mathrm{CH}_{2} \times 2\right), 3.17\left(\mathrm{~s}, 3 \mathrm{H}, \mathrm{NCH}_{3}\right)$, $1.62\left(\mathrm{~s}, 6 \mathrm{H}, \mathrm{CH}_{2} \times 3\right) ;{ }^{13} \mathrm{C}-\mathrm{NMR}\left(\mathrm{DMSO}_{6}\right) \delta: 171.5(\mathrm{C}), 161.7(\mathrm{C}), 140.3(\mathrm{C}), 139.4(\mathrm{C}), 138.6(\mathrm{C})$, $131.1 \mathrm{CH}), 129.5(\mathrm{CH}), 125.2(\mathrm{CH}), 125.1(\mathrm{CH}), 123.5(\mathrm{CH}), 121.9(\mathrm{CH}), 105.3(\mathrm{CH}), 48.3\left(\mathrm{NCH}_{2}\right)$, $30.4\left(\mathrm{NCH}_{3}\right), 25.6\left(\mathrm{CH}_{2}\right), 24.2\left(\mathrm{CH}_{2}\right)$; HRMS m/z: 326.1335 found (calculated for $\mathrm{C}_{18} \mathrm{H}_{19} \mathrm{~N}_{3} \mathrm{OS}$, $[\mathrm{M}+\mathrm{H}]^{+}$requires 326.1322$)$.

(Z)-4-(Benzo[b]thiophen-3-ylmethylene)-2-(3,5-dimethylmorpholino)-1H-imidazol-5(4H)-one (5e). This compound was obtained as bright yellow solid. m.p.: $240-242{ }^{\circ} \mathrm{C}$. IR $(\mathrm{KBr}) \vee\left(\mathrm{cm}^{-1}\right): 3112,1696$, 1640, 1584, 1452; ${ }^{1} \mathrm{H}-\mathrm{NMR}$ (DMSO-d (D) : 11.30 (brs, $\left.1 \mathrm{H}, \mathrm{NH}\right), 8.74$ (s, 1H, Ar-CH), 7.99 (dd, J = 4.1 Hz, $7.8 \mathrm{~Hz}, 2 \mathrm{H}, \mathrm{Ar}-\mathrm{CH}), 7.42-7.37$ (m, 2H, Ar-CH), 6.66 (s, 1H, vinylic proton). 4.01-3.98 (m, 2H, CH), 3.61-3.60 (m, 2H, $\left.\mathrm{CH}_{2}\right), 2.71$ (brs, $\left.2 \mathrm{H}, \mathrm{CH}_{2}\right), 1.13-1.11\left(\mathrm{~m}, 6 \mathrm{H}, \mathrm{CH}_{3} \times 2\right)$; ${ }^{13} \mathrm{C}-\mathrm{NMR}$ (DMSO-d $\left.d_{6}\right)$ $\delta: 172.0(\mathrm{C}), 158.8(\mathrm{C}), 142.3(\mathrm{C}), 139.4(\mathrm{C}), 138.7(\mathrm{C}), 131.2 \mathrm{CH}), 128.7(\mathrm{CH}), 125.1(\mathrm{CH}), 123.5$ $(\mathrm{CH}), 121.7(\mathrm{CH}), 102.7(\mathrm{CH}), 71.3\left(\mathrm{OCH}_{3}\right), 60.1(\mathrm{CH}) 19.0\left(\mathrm{CH}_{3}\right)$; HRMS m/z: 342.1282 found (calculated for $\mathrm{C}_{18} \mathrm{H}_{19} \mathrm{~N}_{3} \mathrm{O}_{2} \mathrm{~S},[\mathrm{M}+\mathrm{H}]^{+}$requires 342.1271).

(Z)-4-(Benzo[b] thiophen-3-ylmethylene)-1-methyl-2-morpholino-1H-imidazol-5(4H)-one (5f). This compound was obtained as bright yellow solid. m.p.: $154-156{ }^{\circ} \mathrm{C}$. IR $(\mathrm{KBr}) \vee\left(\mathrm{cm}^{-1}\right): 1717,1641$, 1561, 1465; ${ }^{1} \mathrm{H}-\mathrm{NMR}\left(\mathrm{DMSO}-d_{6}\right) \delta: 8.79$ (s, $\left.1 \mathrm{H}, \mathrm{Ar}-\mathrm{CH}\right), 8.05(\mathrm{dd}, J=7.45 \mathrm{~Hz}, 7.7 \mathrm{~Hz}, 2 \mathrm{H}, \mathrm{Ar}-\mathrm{CH})$, 7.43-7.38 (m, 2H, Ar-CH), 6.89 (s, $1 \mathrm{H}$, vinylic proton), 3.72-3.70 (m, 4H, $\left.\mathrm{CH}_{2} \times 2\right), 3.62-3.60(\mathrm{~m}$, $\left.4 \mathrm{H}, \mathrm{CH}_{2} \times 2\right), 3.18\left(\mathrm{~s}, 3 \mathrm{H}, \mathrm{NCH}_{3}\right) ;{ }^{13} \mathrm{C}-\mathrm{NMR}\left(\mathrm{DMSO}-d_{6}\right) \delta: 171.2(\mathrm{C}), 161.5(\mathrm{C}), 139.9(\mathrm{C}), 139.3(\mathrm{C})$, $138.6(\mathrm{C}), 130.9(\mathrm{CH}), 130.1(\mathrm{CH}), 125.3(\mathrm{CH}), 125.2(\mathrm{CH}), 123.5(\mathrm{CH}), 121.9(\mathrm{CH}), 106.2(\mathrm{CH})$, $66.1\left(\mathrm{OCH}_{2}\right), 40.3\left(\mathrm{NCH}_{2}\right), 30.2\left(\mathrm{NCH}_{3}\right)$; HRMS m/z: 328.1123 found (calculated for $\mathrm{C}_{17} \mathrm{H}_{17} \mathrm{~N}_{3} \mathrm{O}_{2} \mathrm{~S}$, $[\mathrm{M}+\mathrm{H}]^{+}$requires 328.1114$)$.

(Z)-4-(Benzo[b] thiophen-3-ylmethylene)-1-methyl-2-(3-methylpiperidin-1-yl)-1H-imidazol-5(4H)-one (5g). This compound was obtained as light yellow solid. m.p.: $150-152{ }^{\circ} \mathrm{C}$; IR $(\mathrm{KBr}) v\left(\mathrm{~cm}^{-1}\right): 1709$, 1637, 1561, 1457, 1439; ${ }^{1} \mathrm{H}-\mathrm{NMR}\left(\mathrm{DMSO}-d_{6}\right) \delta: 8.75$ (s, 1H, Ar-CH), 8.05 (dd, J = 7.2 Hz, 7.8 Hz, 
$2 \mathrm{H}, \mathrm{Ar}-\mathrm{CH}), 7.43-7.38(\mathrm{~m}, 2 \mathrm{H}, \mathrm{Ar}-\mathrm{CH}), 6.83$ (s, 1H, vinylic proton), 4.01-3.97 (m, 2H, $\left.\mathrm{CH}_{2}\right), 3.18$ (s, 3H, $\left.\mathrm{NCH}_{3}\right), 3.02-2.9(\mathrm{~m}, 1 \mathrm{H}, \mathrm{CH}), 2.73-2.70\left(\mathrm{~m}, 2 \mathrm{H}, \mathrm{CH}_{2}\right), 1.72-1.69\left(\mathrm{~m}, 4 \mathrm{H}, \mathrm{CH}_{2} \times 2\right), 1.17-1.13$ $\left(\mathrm{m}, 2 \mathrm{H}, \mathrm{CH}_{2}\right), 0.90\left(\mathrm{~d}, J=6.3 \mathrm{~Hz}, 3 \mathrm{H}, \mathrm{CH}_{3}\right) ;{ }^{13} \mathrm{C}-\mathrm{NMR}$ (DMSO-d 6 ) $\delta: 171.5(\mathrm{C}), 161.6(\mathrm{C}), 140.3(\mathrm{C})$, $139.4(\mathrm{C}), 138.6(\mathrm{C}), 131.1(\mathrm{CH}), 129.5(\mathrm{CH}), 125.3(\mathrm{CH}), 125.2(\mathrm{CH}), 123.5(\mathrm{CH}), 121.9(\mathrm{CH}), 105.2$ $(\mathrm{CH}), 54.3\left(\mathrm{CH}_{2}\right), 47.8\left(\mathrm{CH}_{2}\right), 32.7\left(\mathrm{CH}_{2}\right), 31.0\left(\mathrm{CH}_{2}\right), 30.4(\mathrm{CH}), 25.01\left(\mathrm{CH}_{3}\right), 19.3\left(\mathrm{CH}_{3}\right)$; HRMS $\mathrm{m} / \mathrm{z}$ : 340.1485 found (calculated for $\mathrm{C}_{19} \mathrm{H}_{21} \mathrm{~N}_{3} \mathrm{OS},[\mathrm{M}+\mathrm{H}]^{+}$requires 340.1478).

(Z)-4-(Benzo[b]thiophen-3-ylmethylene)-2-thiomorpholino-1H-imidazol-5(4H)-one (5h). This compound was obtained as bright yellow solid. m.p.: 269-271 ${ }^{\circ} \mathrm{C}$; IR $(\mathrm{KBr}) \vee\left(\mathrm{cm}^{-1}\right): 3110,1694,1640,1585$, 1490, 1453; ${ }^{1} \mathrm{H}-\mathrm{NMR}$ (DMSO-d $\left.)\right) \delta: 11.31$ (brs, 1H, NH), 8.70 (s, 1H, Ar-CH), 8.00-7.97 (m, 2H, $\mathrm{Ar}-\mathrm{CH}), 7.42-7.37(\mathrm{~m}, 2 \mathrm{H}, \mathrm{Ar}-\mathrm{CH}), 6.67\left(\mathrm{~s}, 1 \mathrm{H}\right.$, vinylic proton), 3.89 (brs, 4H, $\left.\mathrm{CH}_{2} \times 2\right), 2.68(\mathrm{~s}, 4 \mathrm{H}$, $\left.\mathrm{CH}_{2} \times 2\right) ;{ }^{13} \mathrm{C}-\mathrm{NMR}\left(\mathrm{DMSO}-d_{6}\right) \delta: 172.5(\mathrm{C}), 158.9(\mathrm{C}), 139.5(\mathrm{C}), 138.6(\mathrm{C}), 131.5(\mathrm{C}), 128.7(\mathrm{CH})$, $125.5(\mathrm{CH}), 123.5(\mathrm{CH}), 121.3(\mathrm{CH}), 102.1(\mathrm{CH}), 46.3\left(\mathrm{NCH}_{2}\right), 26.3\left(\mathrm{SCH}_{3}\right) ; \mathrm{HRMS} \mathrm{m} / \mathrm{z}: 330.0737$ found (calculated for $\mathrm{C}_{16} \mathrm{H}_{15} \mathrm{~N}_{3} \mathrm{OS}_{2},[\mathrm{M}+\mathrm{H}]^{+}$requires 330.0729).

(Z)-4-(Benzo[b] thiophen-3-ylmethylene)-2-(4-methylpiperazin-1-yl)-1H-imidazol-5(4H)-one (5i). This compound was obtained as yellow solid. m.p.: 234-236 ${ }^{\circ} \mathrm{C}$; IR $(\mathrm{KBr}) v\left(\mathrm{~cm}^{-1}\right): 3108,1697,1646$, 1591, 1448; ${ }^{1} \mathrm{H}-\mathrm{NMR}\left(\mathrm{DMSO}-d_{6}\right) \delta 11.15$ (brs, 1H, NH), 8.68 (s, 1H, Ar-CH), 7.99 (dd, J = $4 \mathrm{~Hz}, 8 \mathrm{~Hz}$, $2 \mathrm{H}, \mathrm{Ar}-\mathrm{CH}$ ), $7.42-7.37(\mathrm{~m}, 2 \mathrm{H}, \mathrm{Ar}-\mathrm{CH}), 6.64$ (s, 1H, vinylic proton), 3.61 (brs, $\left.4 \mathrm{H}, \mathrm{CH}_{2} \times 2\right), 2.37$ (s, $\left.4 \mathrm{H}, \mathrm{CH}_{2} \times 2\right), 2.19\left(\mathrm{~s}, 3 \mathrm{H}, \mathrm{NCH}_{3}\right) ;{ }^{13} \mathrm{C}-\mathrm{NMR}\left(\mathrm{DMSO}-d_{6}\right) \delta: 172.6(\mathrm{C}), 159.6(\mathrm{C}), 139.3(\mathrm{C}), 138.6(\mathrm{C})$, $131.3(\mathrm{C}), 128.4(\mathrm{CH}), 125.1(\mathrm{CH}), 123.5(\mathrm{CH}), 121.7(\mathrm{CH}), 102.2(\mathrm{CH}), 54.4\left(\mathrm{NCH}_{2}\right), 46.0\left(\mathrm{NCH}_{2}\right)$, 45.4 $\left(\mathrm{NCH}_{3}\right)$; HRMS m/z: 327.1290 found (calculated for $\mathrm{C}_{17} \mathrm{H}_{18} \mathrm{~N}_{4} \mathrm{OS},[\mathrm{M}+\mathrm{H}]^{+}$requires 327.1281).

(Z)-4-(Benzofuran-3-ylmethylene)-1-methyl-2-(piperidin-1-yl)-1H-imidazol-5(4H)-one $\quad$ (5j). This compound was obtained as brown solid. m.p.: $148-150{ }^{\circ} \mathrm{C}$; IR $(\mathrm{KBr}) \vee\left(\mathrm{cm}^{-1}\right): 1713,1649,1556$, 1450; ${ }^{1} \mathrm{H}-\mathrm{NMR}\left(\mathrm{DMSO}-d_{6}\right) \delta: 8.61(\mathrm{~s}, 1 \mathrm{H}, \mathrm{Ar}-\mathrm{CH}), 8.14(\mathrm{~d}, J=7.45 \mathrm{~Hz}, 1 \mathrm{H}, \mathrm{Ar}-\mathrm{CH}), 7.57(\mathrm{~d}$, $J=7.45 \mathrm{~Hz}, 1 \mathrm{H}, \mathrm{Ar}-\mathrm{CH}), 7.34-7.28(\mathrm{~m}, 2 \mathrm{H}, \mathrm{Ar}-\mathrm{CH}), 6.70$ (s, $1 \mathrm{H}$, vinylic proton), $3.53\left(\mathrm{~s}, 4 \mathrm{H}, \mathrm{CH}_{2} \times 2\right)$, $3.15\left(\mathrm{~s}, 3 \mathrm{H}, \mathrm{NCH}_{3}\right), 1.62\left(\mathrm{~s}, 6 \mathrm{H}, \mathrm{CH}_{2} \times 3\right) ;{ }^{13} \mathrm{C}-\mathrm{NMR}\left(\mathrm{DMSO}-d_{6}\right) \delta: 170.8(\mathrm{C}), 161.0(\mathrm{C}), 154.9(\mathrm{C})$, $148.0(\mathrm{CH}), 140.0(\mathrm{C}), 126.6(\mathrm{C}), 125.4(\mathrm{CH}), 123.6(\mathrm{CH}), 121.4(\mathrm{CH}), 117.4(\mathrm{C}), 111.9(\mathrm{CH}), 104.4$ (CH), $48.3\left(\mathrm{NCH}_{2}\right), 30.3\left(\mathrm{NCH}_{3}\right), 25.6\left(\mathrm{CH}_{2}\right), 24.2\left(\mathrm{CH}_{2}\right)$; HRMS m/z: 310.1561 found (calculated for $\mathrm{C}_{18} \mathrm{H}_{19} \mathrm{~N}_{3} \mathrm{O}_{2},[\mathrm{M}+\mathrm{H}]^{+}$requires 310.1557$)$.

(Z)-4-(Benzofuran-3-ylmethylene)-1-methyl-2-morpholino-1H-imidazol-5(4H)-one (5k). This compound was obtained as yellow solid. m.p.: $142-145^{\circ} \mathrm{C}$; IR $(\mathrm{KBr}) v\left(\mathrm{~cm}^{-1}\right)$ : 1708, 1644, 1557, 1452; ${ }^{1} \mathrm{H}-\mathrm{NMR}$ $\left(\right.$ ACETONE- $\left.d_{6}\right) \delta: 8.62(\mathrm{~s}, 1 \mathrm{H}, \mathrm{Ar}-\mathrm{CH}), 8.06(\mathrm{~d}, J=7.00 \mathrm{~Hz}, 1 \mathrm{H}, \mathrm{Ar}-\mathrm{CH}), 7.51(\mathrm{~d}, J=7.00 \mathrm{~Hz}, 1 \mathrm{H}$, $\mathrm{Ar}-\mathrm{CH}), 7.35-7.29(\mathrm{~m}, 2 \mathrm{H}, \mathrm{Ar}-\mathrm{CH}), 6.77$ (s, 1H, vinylic proton), $3.77\left(\mathrm{~s}, 4 \mathrm{H}, \mathrm{OCH}_{2} \times 2\right), 3.60(\mathrm{~s}, 4 \mathrm{H}$, $\left.\mathrm{NCH}_{2} \times 2\right), 3.19\left(\mathrm{~s}, 3 \mathrm{H}, \mathrm{NCH}_{3}\right) ;{ }^{13} \mathrm{C}-\mathrm{NMR}\left(\mathrm{ACETONE}-d_{6}\right) \delta: 170.1(\mathrm{C}), 160.9(\mathrm{C}), 155.0(\mathrm{C}), 148.0$ $(\mathrm{CH}), 139.7(\mathrm{C}), 126.7(\mathrm{C}), 124.8(\mathrm{CH}), 123.1(\mathrm{CH}), 120.4(\mathrm{CH}), 117.1(\mathrm{C}), 111.3(\mathrm{CH}), 105.3(\mathrm{CH})$, $65.9\left(\mathrm{OCH}_{2}\right), 47.5\left(\mathrm{NCH}_{2}\right), 28.9\left(\mathrm{NCH}_{3}\right)$; HRMS m/z: 312.1358 found (calculated for $\mathrm{C}_{17} \mathrm{H}_{17} \mathrm{~N}_{3} \mathrm{O}_{3}$, $[\mathrm{M}+\mathrm{H}]^{+}$requires 312.1350$)$.

(Z)-4-(Benzofuran-3-ylmethylene)-1-methyl-2-thiomorpholino-1H-imidazol-5(4H)-one (5l). This compound was obtained as yellowish solid. m.p.: $157-159{ }^{\circ} \mathrm{C}$; IR $(\mathrm{KBr}) \vee\left(\mathrm{cm}^{-1}\right)$ : 1703, 1649, 1541, 
1450; ${ }^{1} \mathrm{H}-\mathrm{NMR}\left(\mathrm{DMSO}-\mathrm{d}_{6}\right) \delta: 8.62(\mathrm{~s}, 1 \mathrm{H}, \mathrm{Ar}-\mathrm{CH}), 8.14(\mathrm{~d}, J=7.45 \mathrm{~Hz}, 1 \mathrm{H}, \mathrm{Ar}-\mathrm{CH}), 7.58(\mathrm{~d}$, $J=7.45 \mathrm{~Hz}, 1 \mathrm{H}, \mathrm{Ar}-\mathrm{CH}), 7.33-7.29(\mathrm{~m}, 2 \mathrm{H}, \mathrm{Ar}-\mathrm{CH}), 6.76$ (s, 1H, vinylic proton), 3.83-3.81 (m, 4H, $\left.\mathrm{CH}_{2} \times 2\right), 3.15\left(\mathrm{~s}, 3 \mathrm{H}, \mathrm{NCH}_{3}\right), 2.77-2.75\left(\mathrm{~m}, 4 \mathrm{H}, \mathrm{CH}_{2} \times 2\right) ;{ }^{13} \mathrm{C}-\mathrm{NMR}\left(\mathrm{DMSO}-d_{6}\right) \delta: 170.6(\mathrm{C}), 160.9$ (C), $154.9(\mathrm{C}), 148.3(\mathrm{CH}), 139.6(\mathrm{C}), 126.6(\mathrm{C}), 125.5(\mathrm{CH}), 123.7(\mathrm{CH}), 121.4(\mathrm{CH}), 117.3(\mathrm{C})$, $111.9(\mathrm{CH}), 105.5(\mathrm{CH}), 49.9\left(\mathrm{NCH}_{2}\right), 30.2\left(\mathrm{SCH}_{2}\right), 26.5\left(\mathrm{NCH}_{3}\right) ; \mathrm{HRMS} \mathrm{m} / \mathrm{z}: 328.1132$ found (calculated for $\mathrm{C}_{17} \mathrm{H}_{17} \mathrm{~N}_{3} \mathrm{O}_{2} \mathrm{~S}$, $[\mathrm{M}+\mathrm{H}]^{+}$requires 328.1121).

(Z)-4-(Benzofuran-3-ylmethylene)-1-methyl-2-(4-methylpiperazin-1-yl)-1H-imidazol-5(4H)-one (5m). This compound was obtained as a low melting yellow solid. IR $(\mathrm{KBr}) \vee\left(\mathrm{cm}^{-1}\right): 1708,1644,1559$, 1450; ${ }^{1} \mathrm{H}-\mathrm{NMR}\left(\mathrm{DMSO}_{6}+\mathrm{acetone}_{6} \mathrm{~d}_{6}\right) \delta: 9.09$ (s, $\left.1 \mathrm{H}, \mathrm{Ar}-\mathrm{CH}\right), 8.57$ (d, J=7.45 Hz, 1H, Ar-CH), $8.00(\mathrm{~d}, J=7.45 \mathrm{~Hz}, 1 \mathrm{H}, \mathrm{Ar}-\mathrm{CH}), 7.81-7.74(\mathrm{~m}, 2 \mathrm{H}, \mathrm{Ar}-\mathrm{CH}), 7.21$ (s, 1H, vinylic proton), 4.07 (t, $\left.J=4.6 \mathrm{~Hz}, 4 \mathrm{H}, \mathrm{CH}_{2} \times 2\right), 3.66\left(\mathrm{~s}, 3 \mathrm{H}, \mathrm{CH}_{3}\right), 2.93\left(\mathrm{t}, J=4.6 \mathrm{~Hz}, 4 \mathrm{H}, \mathrm{CH}_{2} \times 2\right), 2.68\left(\mathrm{~s}, 3 \mathrm{H}, \mathrm{CH}_{3}\right)$; ${ }^{13} \mathrm{C}-\mathrm{NMR}\left(\mathrm{DMSO}_{6}+\mathrm{ACETONE}_{6} \mathrm{~d}_{6}\right) \delta: 170.9(\mathrm{C}), 161.4(\mathrm{C}), 155.4(\mathrm{C}), 148.5(\mathrm{CH}), 140.3(\mathrm{C})$, $127.1(\mathrm{C}), 125.6(\mathrm{CH}), 123.8(\mathrm{CH}), 121.4(\mathrm{CH}), 117.7(\mathrm{C}), 112.0(\mathrm{CH}), 105.4(\mathrm{CH}), 54.8\left(\mathrm{CH}_{2}\right), 47.6$ $\left(\mathrm{CH}_{2}\right), 46.2\left(\mathrm{CH}_{3}\right), 30.2\left(\mathrm{CH}_{3}\right)$; HRMS m/z: 325.1670 found (calculated for $\mathrm{C}_{18} \mathrm{H}_{20} \mathrm{~N}_{4} \mathrm{O}_{2},[\mathrm{M}+\mathrm{H}]^{+}$ requires 325.1666$)$.

(Z)-4-(Benzofuran-3-ylmethylene)-2-(3,5-dimethylmorpholino)-1-methyl-1H-imidazol-5(4H)-one (5n). This compound was obtained as bright yellow solid. m.p.: $137-140{ }^{\circ} \mathrm{C}$; IR $(\mathrm{KBr}) v\left(\mathrm{~cm}^{-1}\right)$ : 1703,1641 ,

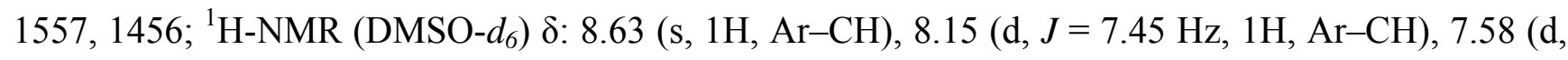
$J=7.45 \mathrm{~Hz}, 1 \mathrm{H}, \mathrm{Ar}-\mathrm{CH}), 7.33-7.28(\mathrm{~m}, 2 \mathrm{H}, \mathrm{Ar}-\mathrm{CH}), 6.74(\mathrm{~s}, 1 \mathrm{H}$, vinylic proton), 3.98 (d, $J=12.6 \mathrm{~Hz}$, 2H, $\left.\mathrm{CH}_{2}\right), 3.71-3.69\left(\mathrm{~m}, 2 \mathrm{H}, \mathrm{CH}_{2}\right), 3.18\left(\mathrm{~s}, 3 \mathrm{H}, \mathrm{CH}_{3}\right), 2.78-2.75(\mathrm{~m}, 2 \mathrm{H}, \mathrm{CH}), 1.13$ (d, J = 12.6 Hz, $\left.6 \mathrm{H}, \mathrm{CH}_{3} \times 2\right) ;{ }^{13} \mathrm{C}-\mathrm{NMR}\left(\mathrm{DMSO}_{\mathrm{d}}\right.$ ) $\delta: 170.7(\mathrm{C}), 160.4(\mathrm{C}), 154.9(\mathrm{C}), 148.3(\mathrm{CH}), 139.6(\mathrm{C}), 126.6$ (C), $125.8(\mathrm{CH}), 123.6(\mathrm{CH}), 121.4(\mathrm{CH}), 117.3(\mathrm{C}), 112.0(\mathrm{CH}), 105.3(\mathrm{CH}), 71.2\left(\mathrm{OCH}_{2}\right), 52.2(\mathrm{CH})$, $30.2\left(\mathrm{CH}_{3}\right), 19.0\left(\mathrm{CH}_{3}\right)$; HRMS m/z: 340.1671 found (calculated for $\mathrm{C}_{19} \mathrm{H}_{21} \mathrm{~N}_{3} \mathrm{O}_{3},[\mathrm{M}+\mathrm{H}]^{+}$requires $340.1663)$.

\section{Conclusions}

In summary we have successfully developed a synthetic method that provides ready access to novel biologically important benzo[b]thiophene and benzofuran based thiohydantoin derivatives. We are currently investigating the synthesis of a number of other thiohydantoin-based drug molecules by this method. A detailed biological activity study (antibacterial, antifungal, anticancer and neuroprotective kinase inhibitor activity) of these important compounds is being carried out. Preliminary results indicate that many of the thiohydantoins exhibit excellent neuroprotective properties.

\section{Acknowledgments}

The authors are grateful to NIH (1RC2NS064950) for generous financial support. 


\section{References and Notes}

1. Spinks, A.; Waring, W.S. Anticonvulsant drugs. Prog. Med. Chem. 1963, 3, 261-331.

2. Khodair, A.I.; El-Subbagh, H.I.; El-Emam, A.A. Synthesis of certain 5-substituted 2-thiohydantoin derivatives as potential cytotoxic and antiviral agents. Boll Chim Farm 1997, 136, 561-567.

3. Al-Obaid, A.A.; El-Subbagh, H.I.; Khodair, A.I.; El-Mazar, M.M. 5-Substituted-2-thiohydant-oin analogs as a novel class of antitumor agent. Anti-Cancer Drugs 1996, 7, 873-880.

4. El-Barbary, A.A.; Khodair, A.I.; Pedersen, E.B.; Nielsen, C. S-Glucosylated hydantoins as new antiviral agents. J. Med. Chem. 1994, 37, 73-77.

5. Van Coillie, E.; Van Damme, J.; Opdenakker, G. The MCP/eotaxin subfamily of CC chemokines. Cytokine Growth Factor Rev. 1999, 10, 61-86.

6. Collin, M.; Thiemermann, C. The PPAR-gamma ligand 15-deoxy(delta12,14) prostaglandin J2 reduces the liver injury in endotoxic shock. Eur. J. Pharmacol. 2003, 29, 257-258.

7. Ohata, M.; Suzuki, H.; Sakamoto, K.; Hasimoto, K.; Nakajima, H.; Yamauchi, M.; Hokkyo, K.; Yamada, H.; Toda, G. Pioglitazone prevents acute liver injury induced by ethanol and lipopolysaccharide through the suppression of tumor necrosis factor-alpha. Alcohol. Clin. Exp. Res. 2004, 28, 139S-144S.

8. Ankati, H.; Akubathini, S.K.; D'Mello, S.R.; Biehl, E.R. Synthesis of 2-benzylidene and 2-hetarylmethyl derivatives of 2h-1,4-benzoxazin-3-(4h)-ones as neuroprotecting agents. Syn. Commun. 2010, 40, 2364-2376.

9. Wang, L.; Ankati, H.; Akubathini, S.K.; Balderamos, M.; Storey, C.A.; Patel, A.V.; Kretzschmar, D.; Biehl, E.R.; D'Mello, S.R. Identification of novel 1,4-benzoxazine compounds that are protective in tissue culture and in vivo models of neurodegeneration. J. Neurosci. Res. 2010, 88, 1970-1984.

10. Balderamos, M.; Ankati, H.; Akubathini, S.K.; Patel, A.V.; Kamila, S.; Mukherjee, C.; Wang, L.; Biehl, E.R.; D'Mello, S.R. Synthesis and structure-activity relationship studies of 3-substituted indolin-2-ones as effective neuroprotective agents. Exp. Biol. Med. (Maywood, N.J., USA) 2008, 233, 1395-1399.

11. Nielek, S.; Lesiak, T. Chemistry of thiazole, I. Synthesis and properties of 2,3,5,6-tetrahydro-6-(3methylbenzofuran-2-yl)imidazo[2,1-b]thiazole. Chem. Ber. 1982, 115, 1247-1251.

12. Zaidlewicz, M.; Chechlowska, A.; Prewysz- Kwinto, A.; Wojtczak, A. Enatntioselective synthesis of 2-and 3-benzofuryl $\beta$-amino alcohols. Heterocycles 2001, 55, 569-577.

13. Sun, Y.; Gao, L.P.; Ding, M.W. Facile synthesis of 2-alkylthio-3-alkyl-5-phenylmethylidene-4Himidazol-4-ones. Syn. Commun. 2006, 36, 1185-1191.

14. Kiec-Kononowicz, K.; Szymanska, E.; Motyl, M.; Holzer, W.; Bialecka, A.; Kasprowicz, A. Synthesis, spectral, and antimicrobial properties of 5-chloroarylidene aromatic derivatives of imidazolin-4-one. Pharmazie 1998, 53, 680-684.

Sample Availability: Samples of the compounds $\mathbf{5 a - n}$ are available from the authors.

(C) 2011 by the authors; licensee MDPI, Basel, Switzerland. This article is an open access article distributed under the terms and conditions of the Creative Commons Attribution license (http://creativecommons.org/licenses/by/3.0/). 